\title{
Comparison between intermediate and severe coronary stenoses and clinical outcomes of an OCT-guided PCl strategy
}

\author{
Luca Di Vito ${ }^{a}$, Maria Alberta Cattabiani ${ }^{b}$, Giulia Paoletti ${ }^{a}$, Joo Heung Yoon ${ }^{c}$, \\ Alberto Chisari ${ }^{a}$, Mario Gramegna ${ }^{d}$, Francesco Versaci ${ }^{d}$, Fausto Castriota ${ }^{\mathrm{e}}$ \\ and Francesco Prati ${ }^{\mathrm{a}}$
}

\begin{abstract}
Aims We compared optical coherence tomography (OCT) features of intermediate and severe coronary stenoses in patients with stable angina and acute coronary syndrome (ACS), and tested the clinical impact of an OCT-based strategy for treating intermediate stenoses.
\end{abstract}

Methods The study enrolled 135 consecutive patients with either ACS or stable angina and a single de-novo coronary stenosis. Patients were divided into two groups: intermediate stenosis defined as quantitative coronary angiography percentage narrowing less than $70 \%$, or presence of angiographic vessel haziness and severe stenosis with percentage narrowing more than $70 \%$. OCT was performed to assess features of plaque vulnerability and to measure the minimal lumen area. We also appraised the 12-month rate of major adverse event (MACE) of an OCT-guided strategy of percutaneous coronary intervention (PCl) based on the presence of thrombus and/or minimal lumen area less than $3.0 \mathrm{~mm}^{2}$.

Results Fifty-six patients had intermediate stenoses, whilst 79 had severe stenoses. In the 'intermediate stenosis group', patients with stable angina had a lower asymmetric index $(P=0.02)$ and a greater calcific arc $(P=0.0001)$. In the 'severe stenosis group', intermediate lesions of patients with ACS exhibited a greater lipid arc as compared with

\section{Introduction}

Intermediate stenosis is often defined as a non-obstructive vessel narrowing comprised between 30 and $70 \%$ at quantitative coronary angiography (QCA). They represent a grey zone for interventional cardiologists and often lead to controversies regarding the need to perform percutaneous coronary intervention (PCI). ${ }^{1}$ Frequency domain optical coherence tomography (FD-OCT) is a high-resolution $(20 \mu \mathrm{m})$ intravascular imaging modality that allows a detailed analysis of the intermediate plaques. FD-OC'T accurately identifies lumen area contour and depicts anatomical features indicative of plaque vulnerability or instability, such as thin cap fibroatheroma (TCFA) or coronary thrombus. ${ }^{2,3}$

In this study, we sought to assess the FD-OCT-based plaque features of intermediate coronary stenosis at angiography, in the clinical settings of stable angina patients with stable angina $(P=0.03)$. A higher prevalence of thin cap fibroatheroma was seen in patients with ACS of both groups. The incidence of MACE was not significantly different between patients with an intermediate stenosis who received $\mathrm{PCl}$ vs. optimal medical therapy on the basis of OCT findings $(P=0.26)$.

\begin{abstract}
Conclusions Intermediate coronary stenoses showed distinctive OCT-based features according to the initial clinical presentation. The adoption of an OCT-guided PCI strategy, based on the presence of coronary thrombus and significant vessel narrowing, led to encouraging results.
\end{abstract}

J Cardiovasc Med 2016, 17:361-367

Keywords: intra-coronary imaging, optical coherence tomography, plaque rupture

aSan Giovanni Addolorata Hospital and CLI foundation, Rome, ${ }^{\text {bDivision of }}$ Cardiology, Parma Hospital, Parma, Italy, 'Beth Israel Deaconess Medical Center, Harvard Medical School, Boston, Massachusetts, USA, 'Ospedale 'F. Veneziale', Isernia and ${ }^{\mathrm{e} V}$ illa Maria Cecilia Hospital - Cotignola, Italy

Correspondence to Francesco Prati, Interventional Cardiology Unit, San Giovanni Addolorata Hospital, Via dell'Amba Aradam 8, 00184 Rome, Italy Tel: +39 0677055330; fax: +39 0696527673;

e-mail: fprati@hsangiovanni.roma.it

Received 18 September 2014 Revised 6 January 2015 Accepted 15 February 2015

and acute coronary syndrome (ACS). In addition, we addressed the clinical utility of a strategy of PCI guidance of intermediate stenoses, based on the presence of coronary thrombus and/or a minimal lumen area (MLA) less than $3.0 \mathrm{~mm}^{2}$.

\section{Methods}

\section{Patient populations}

A total of 135 consecutive patients were retrospectively included in the study, from December 2010 until March 2012. Among these, 56 patients had an angiographically defined intermediate stenosis, and 79 had a severe stenosis.

Inclusion criteria were as follows: for the intermediate stenosis group, presence of a single de-novo stenosis with a percentage stenosis less than $70 \%$ by QCA or the presence of angiographic vessel haziness defined as a 
defect in the contrast filling of the coronary artery; for the severe lesion group, presence of a single de-novo stenosis more than $70 \%$ by QCA without haziness at angiography; presence of either stable angina or ACS on hospital admission; and successful baseline assessment with coronary OCT. Stable angina patients had to have signs of induced myocardial ischaeamia at non-invasive stress tests, ${ }^{4,5}$ but there was no change in frequency, duration, or intensity of angina symptoms before hospital admission. ACS patients included unstable angina, ${ }^{5}$ non-STsegment elevation myocardial infarction (NSTEMI), ${ }^{6}$ and ST-segment elevation myocardial infarction (STEMI). ${ }^{7}$

Exclusion criteria were as follows: left main stenosis; ostial stenosis; presence of overlapping vessels; a large side-branch (greater than $2 \mathrm{~mm}$ in diameter); in-stent restenosis or thrombosis; previous coronary artery bypass surgery; and stenosis location in a distal segment of the coronary tree that could not be imaged with OCT.

The study was conformed by the Declaration of Helsinki on human research, and informed consent was obtained from each patient.

\section{Quantitative coronary angiographic analysis}

Quantitative coronary angiography analysis was performed with a validated edge-detection system (CAAS Version 5.9, Pie Medical Imaging, Maastricht, the Netherlands). Reference vessel diameter, minimal lumen diameter, percentage of diameter stenosis, and stenosis length were measured in diastolic frames from orthogonal projections according to validated protocols ${ }^{8}$ for both intermediate and severe stenoses.

\section{Frequency domain optical coherence tomography procedure and analysis of optical coherence tomography images}

Frequency domain optical coherence tomography was performed with non-occlusive techniques, as previously described. ${ }^{9}$ After intra-coronary injection of nitroglycerin $200 \mu \mathrm{g}$, OCT images were acquired with a commercially available C7 system (LightLab Imaging Inc/St Jude Medical, Westford, Massachusetts, USA) after positioning of the C7 Dragonfly OCT catheter (LightLab Imaging Inc/St Jude Medical) distal to the target segment.

During OCT image acquisition, angiographic contrast media were injected through the guiding catheter with an automated power injector. The OCT pull-back speed was set at $20 \mathrm{~mm} / \mathrm{s}$, and the OCT images were calibrated adjusting the Z-offset. All OCT images were analysed offline in a centralized core lab (Rome Heart Research, Rome, Italy) using a dedicated OCT consol (LightLab Imaging Inc/St Jude Medical). The images were read by two readers (L.D.V. and F.P.) who were blinded to the protocol.
Frequency domain OCT analysis was then performed at the MLA site (MLA site analysis) and along the entire plaque (plaque analysis). At the MLA site, the following measurements were obtained: lumen area, minimal and maximal diameters, mean diameter, and asymmetric index, calculated as [(maximal diameter-minimal diameter)/maximal diameter]. An asymmetric index greater than 0.3 was indicative of asymmetric luminal narrowing. Plaque composition (lipid, fibrotic, and calcific), circumferential extension of lipid and calcium arcs, and a fibrous cap thickness were also measured as previously described. ${ }^{2,3}$

Plaque analysis was performed along the entire plaque length in every cross-section. The analysis included presence of TCFA, plaque rupture, thrombus, ${ }^{10,11}$ micro-vessels, ${ }^{12}$ and superficial calcified nodules. ${ }^{13}$ Measurements of plaque length cap thickness and lipid quadrants were also obtained.

\section{Definitions of plaque components}

A plaque was defined as lipid-rich if it contained two or more lipid quadrants. TCFA was defined as a lipid-rich plaque with a fibrous cap thickness of $65 \mu \mathrm{m}$ or less. ${ }^{11,14,15}$ A micro-channel was defined as a no-signal tubulo-luminal structure without a connection to the vessel lumen, which was recognized on at least three consecutive crosssectional OCT images. ${ }^{15}$ Thrombus was identified as an irregular-shaped mass with dorsal shadowing protruding into the vessel lumen or discontinuation from the surface of the vessel wall. ${ }^{10}$

\section{Optical coherence tomography-guided treatment strategy for intermediate stenoses}

Patients with an intermediate stenosis were treated with PCI if OCT showed a coronary intraluminal thrombus ${ }^{2}$ and/or a MLA less than $3.0 \mathrm{~mm}^{2}$, according to the procedural standards applied in our catheterization laboratory. ${ }^{16}$ The remaining patients received optimal medical therapy (OMT) only.

\section{Definitions of follow-up clinical endpoints}

Information about baseline characteristics and clinical events were obtained at 3, 6, 9, and 12 months with ambulatory visits, telephone contacts, or hospital medical records in case of coronary events.

The primary composite clinical endpoint included cardiovascular death, myocardial infarction (MI), and repeated revascularization. Secondary endpoints were each single endpoint of the primary composite (cardiovascular death, MI, or repeated revascularization). In detail, MI during follow-up was defined as an increase in cardiac biomarkers, with at least one value above the 99th percentile, along with at least one of the following: symptoms of ischaemia; new or presumed new significant ST-segment or T-wave changes, or new left bundle branch block; development of new pathologic $Q$ waves 
on an electrocardiogram; imaging evidence of new loss of viable myocardium or new regional wall motion abnormality; or identification of an intra-coronary thrombus by angiography or autopsy. ${ }^{17}$ Repeated revascularization was defined as any repeated PCI to treat stent restenosis, or new PCI to treat intermediate stenosis for which intervention was deferred.

\section{Statistical analysis}

Normal distribution was assessed by the KolmogorovSmirnov test. Continuous variables were expressed as means $\pm \mathrm{SD}$, and assessed with Student's $t$ test; categorical variables were expressed as proportions and assessed with Fisher's exact test. value less than 0.05 was required for statistical significance. For statistical analysis, SPSS 17.0 (SPSS, Inc., Chicago, Illinois, USA) was used.

\section{Results}

\section{Baseline patient characteristics}

Baseline clinical features are shown in Table 1. Fifty-six patients out of 135 entered the intermediate stenosis group. Thirty patients $(53 \%)$ had a stable angina, whilst 26 had an ACS (47\%). Of these, 14 had NSTEMI/ unstable angina, and 12 had STEMI. The severe stenosis group included 79 patients. Among them, 38 patients $(48 \%)$ had stable angina, whereas the remaining $41(52 \%)$ had ACS, with NSTEMI/unstable angina diagnosed in 21 patients, and STEMI in 20 patients.

\section{Angiographic findings}

Baseline angiographic findings are summarized in Table 2.

In the intermediate stenosis group, baseline \%diameter stenosis was $53 \pm 15 \%$ in the stable angina group and $56 \pm 15 \%$ in the ACS group $(P=0.4)$. The prevalence of vessel angiographic haziness was similar in the groups with stable angina and ACS. In the severe stenosis group, $\%$ diameter stenosis was $70 \pm 11 \%$ in the stable angina group and $72 \pm 12 \%$ in the ACS group $(P=0.3)$.

\section{Optical coherence tomography findings}

\section{Minimal lumen area site}

In the intermediate stenosis group, patients with stable angina had larger MLA $\left(3.5 \pm 1.5\right.$ vs. $2.7 \pm 1.3 \mathrm{~mm}^{2}$; $P=0.04)$, but exhibited a lower asymmetric index (Fig. 1a) as compared with ACS (Fig. 1b) $(0.24 \pm 0.13$ vs. $0.33 \pm 0.15 ; P=0.02)$. Table 3 depicts plaque composition in the stable angina and the ACS group. In the presence of calcific MLA, the calcium arc was significantly higher in stable angina group (Fig. 1c) as compared with the ACS group $\left(242 \pm 57\right.$ vs. $\left.114 \pm 35^{\circ} ; P=0.0001\right)$.

In the severe stenosis group, no difference was noted in the MLA between the ACS and the stable angina group $\left(2.6 \pm 0.8\right.$ vs. $\left.2.3 \pm 1.2 \mathrm{~mm}^{2} ; P=0.22\right)$. Consistently, the asymmetric index did not differ between the two groups $(P=0.94)$. Lipid plaques were more commonly imaged in the ACS compared with the stable angina group $(P=0.03)$.

\section{Overall plaque}

In the intermediate stenosis group, plaque length was not significantly different in patients with ACS as compared with patients with stable angina $(P=0.67)$. TCFA (Fig. 2a) and thrombus (Fig. 2b) were significantly more common in ACS patients (26.9 vs. 6.7\%; $P=0.04$ and 38.5 vs. $10 \% ; P=0.01$, respectively). However, no difference was found between the two groups in the incidence of plaque rupture $(30.8$ vs. $16.7 \% ; P=0.21)$, as depicted in Table 4.

In the severe stenosis group, plaque length was significantly greater in patients with ACS as compared with patients with stable angina $(14.2 \pm 5.4$ vs. $10.6 \pm 4.4 \mathrm{~mm}$; $P=0.002)$. Presence of TCFA, thrombus, and ruptured plaque was significantly more common in the ACS group (68 vs. $44 \% ; P=0.03$, 48 vs. $0 \% ; P=0.0001$ and 75.6 vs. $31.6 \% ; P=0.001$, respectively), whereas calcified nodules were more frequently found in patients with stable angina (89.5 vs. $68.3 \% ; P=0.02)$.

Table 1 Baseline clinical features

\begin{tabular}{|c|c|c|c|c|c|c|}
\hline & \multicolumn{3}{|c|}{ Intermediate lesions (56) } & \multicolumn{3}{|c|}{ Severe lesions (79) } \\
\hline & SA (30) & ACS (26) & $P$ & SA (38) & $\operatorname{ACS}(41)$ & $P$ \\
\hline Age [years (SD)] & $68.8( \pm 9.1)$ & $64.3( \pm 9.7)$ & 0.17 & $71.4( \pm 5.3)$ & $70.2( \pm 12)$ & 0.73 \\
\hline Male sex $[n(\%)]$ & $19(63)$ & $17(65)$ & 0.87 & $22(60)$ & $24(58)$ & 0.95 \\
\hline STEMI $[n(\%)]$ & & $12(46)$ & & & $20(48)$ & \\
\hline NSTEMI $[n(\%)]$ & & $14(54)$ & & & $21(52)$ & \\
\hline Hypertension [n (\%)] & $24(80)$ & $19(73)$ & 0.7 & $22(58)$ & $31(76)$ & 0.08 \\
\hline Diabetes mellitus [ $n(\%)]$ & $14(46)$ & 9 (35) & 0.46 & $12(31)$ & $16(39)$ & 0.63 \\
\hline Current smoker [n (\%)] & $8(27)$ & $12(46)$ & 0.15 & $14(37)$ & $12(29)$ & 0.41 \\
\hline Dyslipidaemia [n (\%)] & $23(77)$ & $19(73)$ & 0.80 & $37(97)$ & $25(61)$ & 0.001 \\
\hline Family history [n (\%)] & $4(13)$ & $11(42)$ & 0.002 & $8(21)$ & $7(17)$ & 0.73 \\
\hline Previous PCI [n (\%)] & $14(46)$ & $10(38)$ & 0.44 & $16(42)$ & $13(32)$ & 0.33 \\
\hline
\end{tabular}

ACS, acute coronary syndrome; NSTEMI, non-ST-segment elevation myocardial infarction; PCI, percutaneous coronary intervention; SA, stable angina; STEMI, STsegment elevation myocardial infarction. 
Table 2 Angiographic findings

\begin{tabular}{|c|c|c|c|c|c|c|}
\hline & \multicolumn{3}{|c|}{ Intermediate lesions (56) } & \multicolumn{3}{|c|}{ Severe lesions (79) } \\
\hline & SA (30) & $\operatorname{ACS}(26)$ & $P$ & SA (38) & ACS (41) & $P$ \\
\hline Lesion length [mm (SD)] & $6.0(3.0)$ & $7.9(3.0)$ & 0.03 & $8.3(6.5)$ & $8.9(7.4)$ & 0.2 \\
\hline Obstruction diameter [mm (SD)] & $1.9(0.6)$ & $1.8(0.5)$ & 0.60 & $0.8(0.4)$ & $0.6(0.3)$ & 0.41 \\
\hline Reference diameter [mm (SD)] & $2.9(0.7)$ & $2.9(0.7)$ & 0.84 & $2.9(0.4)$ & $2.5(0.6)$ & 0.01 \\
\hline Percentage diameter stenosis [\% (DS)] & $53.1(15.0)$ & $56.1(15.3)$ & 0.47 & $70.2(11.5)$ & $72.7(12.0)$ & 0.32 \\
\hline Vessel haziness $[n(\%)]$ & $8(27 \%)$ & $9(35 \%)$ & 0.52 & 0 & 0 & \\
\hline
\end{tabular}

ACS, acute coronary syndrome; DS, diameter stenosis; SA, stable angina.

\section{Optical coherence tomography findings in the presence of angiographic haziness}

In the intermediate stenosis group, angiography revealed the presence of haziness in eight cases with stable angina $(27 \%)$ vs. $9(35 \%)$ with ACS.

Optical coherence tomography showed that, in the presence of stable angina, vessel angiographic haziness was due to calcified components in $87.5 \%$ and thrombus in $12.5 \%$. On the contrary, in the presence of ACS, angiographic haziness was due to calcium in $33.3 \%$ and thrombus in $66.6 \%$.

\section{Optical coherence tomography-guided treatment strategy of patients with intermediate stenoses}

Intermediate stenoses were treated with PCI in 33 out of the 56 patients $(59 \%)$. PCIs were done more often in the ACS group, although the difference was not statistically significant (69.2 vs. $50 \%$, respectively; $P=0.14$ ). PCI was performed in all 13 stenoses, showing a coronary thrombus, and in 10 out of 13 ruptured plaques $(77 \%)$.

The median follow-up duration was 352 days (SD 29 days). One patient in the PCI-treated group died due to MI, whilst in the OMT group, one case with non-fatal MI and a second one with repeated revascularization were reported. As a result, the primary endpoint of the composite of cardiovascular death, MI, and repeated revascularization was not significantly different between patients with an intermediate stenosis who received PCI vs. OMT on the base of OC'T findings $(P=0.26$; Table 5).

\section{Discussion}

In the present study, we investigated OCT features of plaque characteristics of angiographically defined, intermediate coronary stenoses on the basis of vessel narrowing or vessel angiographic haziness.

In the presence of an intermediate coronary stenosis, patients with stable angina or ACS showed different features at the MLA site. In the stable angina group, symmetric vessel narrowing with large calcification was typically observed, whereas an asymmetric vessel narrowing was more commonly imaged in ACS. Furthermore, a higher incidence of thrombus was found in the ACS group.

An intermediate stenosis is a common finding of coronary angiography that often leads to controversies regarding the treatment options to be applied. The use of intra-coronary diagnostic modalities including functional assessment of target stenosis (i.e. FFR) ${ }^{18-20}$

Fig. 1

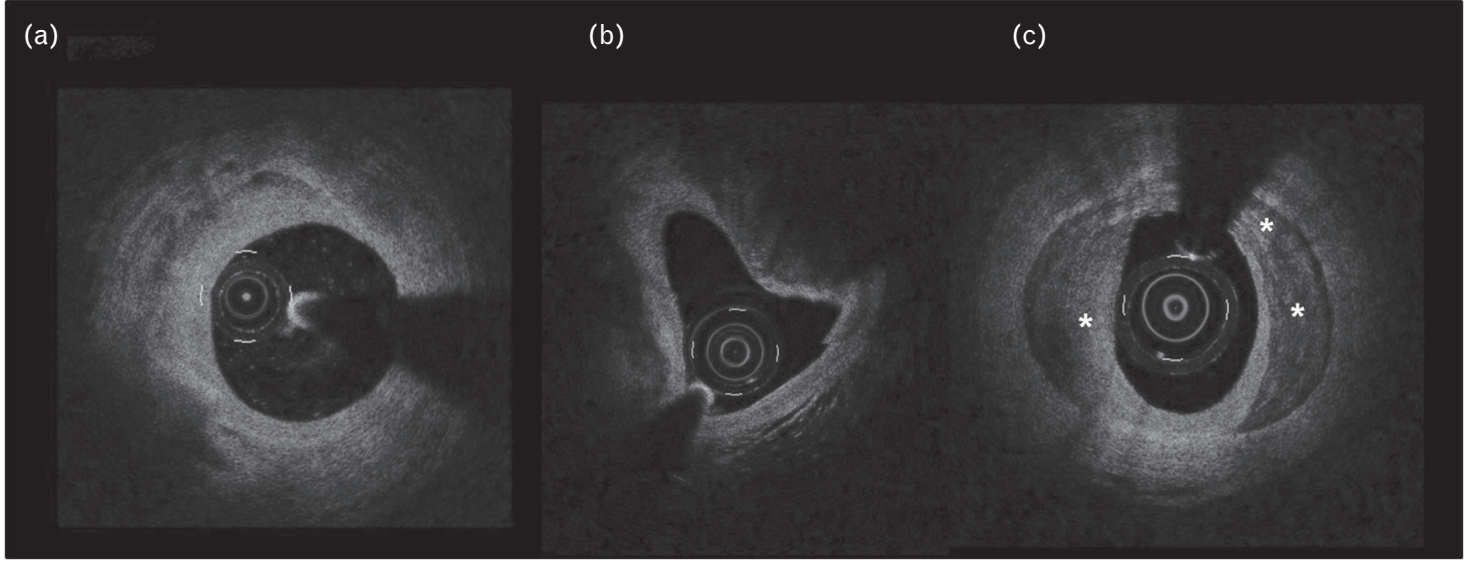

Optical coherence tomography-based characteristics of intermediate stenoses found at the MLA site. Panel a: A symmetric vessel narrowing characterized by a concentric thickness imaged in a patient with stable angina. Panel b: An asymmetric vessel narrowing with an irregular luminal contour imaged in a patient with ACS. Panel c: A calcified plaque with a large calcium plate (asterisks) extending deep in the media layer for 310 degrees imaged in a patient with stable angina. ACS, acute coronary syndrome; MLA, minimal lumen area. 
Table 3 Optical coherence tomography results at the minimal lumen area site

\begin{tabular}{|c|c|c|c|c|c|c|}
\hline & \multicolumn{3}{|c|}{ Intermediate lesions (56) } & \multicolumn{3}{|c|}{ Severe lesions (79) } \\
\hline \multicolumn{7}{|l|}{ Measurement findings } \\
\hline Mean diameter [mm (SD)] & $2.0(0.4)$ & $1.7(0.4)$ & 0.01 & $1.8(0.3)$ & $1.6(0.2)$ & 0.03 \\
\hline Minimal diameter [mm (SD)] & $1.7(0.3)$ & $1.4(0.5)$ & 0.01 & $1.4(0.3)$ & $1.3(0.4)$ & 0.20 \\
\hline Maximal diameter [mm (SD)] & $2.3(0.5)$ & $2.1(0.4)$ & 0.04 & $2.1(0.4)$ & $1.9(0.2)$ & 0.02 \\
\hline Fibrous plaque $[n(\%)]$ & $3(10)$ & $1(3.8)$ & & $6(14.6)$ & 0 & \\
\hline Calcified plaque $[n(\%)]$ & $10(33)$ & $8(30.8)$ & & $2(4.9)$ & $4(10.5)$ & \\
\hline Lipid arc [degrees (SD)] & $193.3(73.2)$ & $216.5(61.4)$ & 0.33 & $230.6(91.8)$ & $301.1(79.2)$ & 0.01 \\
\hline Calcific arc [degrees (SD)] & $242.0(57.8)$ & $114.7(35.1)$ & 0.0001 & $96.1(31.1)$ & $107.4(45.1)$ & 0.78 \\
\hline Fibrous cap thickness [ $\mu \mathrm{m}(\mathrm{SD})]$ & $91(53)$ & $88(65)$ & 0.83 & $102(34)$ & $49(23)$ & 0.01 \\
\hline
\end{tabular}

ACS, acute coronary syndrome; MLA, minimal lumen area; SA, stable angina.

is a valid option to decide whether to proceed with angioplasty or to defer interventions.

In the present study, we showed that intermediate stenoses with a vessel narrowing comprised between 30 and $70 \%$ by QCA have distinctive OCT -derived features that are related to the clinical presentation (stable angina or ACS). Currently, coronary angiography is the technique of choice to assess coronary atherosclerosis; however, it cannot depict the plaque beyond the lumen, ${ }^{21}$ and as a consequence is unable to provide information on plaque vulnerability. ${ }^{2}$

Previous angiographic studies stressed that plaque rupture can occur at the site of non-severely stenotic lesion. $^{22-24}$ More recently, in-vivo studies using both intravascular imaging techniques or ex-vivo autoptycal studies showed that culprit plaques causing sudden death or MI tend to have a larger plaque burden with a thinner fibrous cap and a smaller lumen area, compared with stable or non-ruptured plaques. ${ }^{25-27}$

In the present study, we identified a high prevalence of TCFA in intermediate stenoses visualized in patients with ACS. This conclusion is in line with other OCT studies pointing out that TCFA is often found in nonseverely stenotic lesions.

Previous studies have also shown that in the setting of ACS ruptured plaque can be imaged in both culprit and non-culprit coronary vessels. ${ }^{28,29}$ Consistently with the previous data, in the present study, we found ruptured plaques at the site of intermediate narrowing in the presence of both stable angina or ACS.

Fig. 2

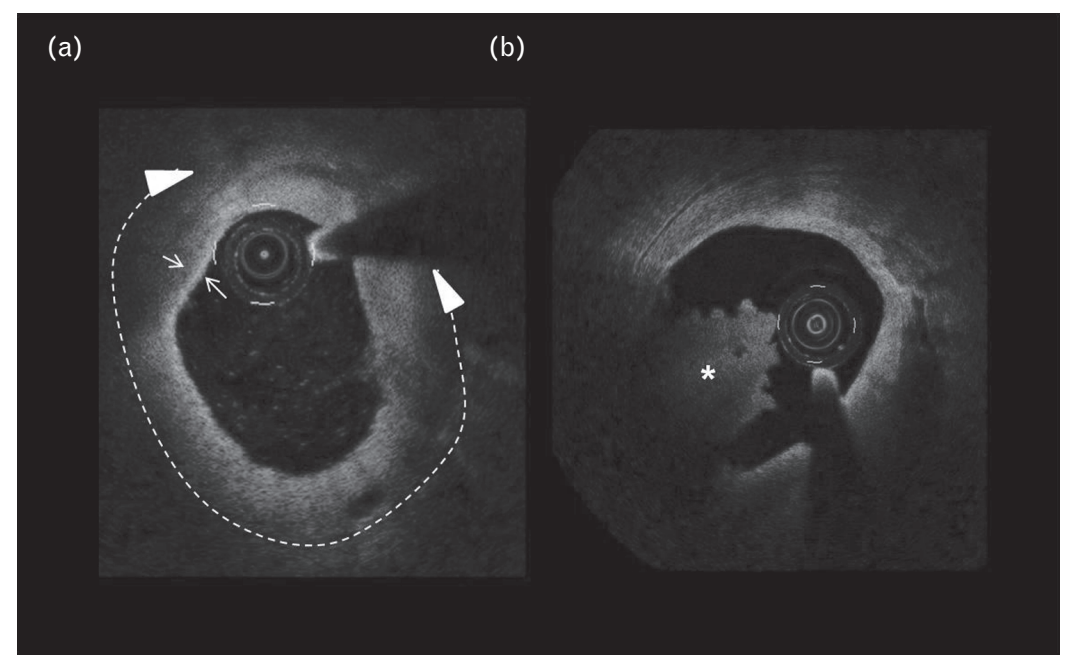

Optical coherence tomograph-based characteristics of intermediate stenoses found along the entire plaque. Panel a: TCFA is imaged in a patient with ACS. A large lipid pool (dashed line) extending for $270^{\circ}$ exists, and is partially covered by a thin fibrous cap (approximately $60 \mu \mathrm{m}$ ) at 10 o'clock (arrows). Panel b: A coronary thrombus is imaged in another ACS patient. Thrombus is imaged as an irregular mass protruding into the lumen (asterisk). It shows a typical shadowing effect due to a high attenuation. ACS, acute coronary syndrome; TCFA, thin cap fibroatheroma. 
Table 4 Optical coherence tomography results along the entire imaged plaque

\begin{tabular}{|c|c|c|c|c|c|c|}
\hline & \multicolumn{3}{|c|}{ Intermediate lesions (56) } & \multicolumn{3}{|c|}{ Severe lesions (79) } \\
\hline & SA (30) & ACS (26) & $P$ & SA (38) & ACS (41) & $P$ \\
\hline TCFA [n (\%)] & $2(6.7)$ & $7(26.9)$ & 0.04 & $17(44.7)$ & $28(68.3)$ & 0.03 \\
\hline Ruptured plaque [n (\%)] & $5(16.7)$ & $8(30.8)$ & 0.21 & $12(31.6)$ & $31(75.6)$ & 0.001 \\
\hline Calcified nodule $[n(\%)]$ & $2(6.7)$ & 9 (34.6) & 0.09 & 34 (89.5) & $28(68.3)$ & 0.02 \\
\hline Micro-vessel $[n(\%)]$ & $4(13.3)$ & 0 & 0.05 & $33(86.8)$ & $35(85.4)$ & 0.85 \\
\hline Thrombus $[n(\%)]$ & $3(10)$ & $10(38.5)$ & 0.01 & 0 & $20(48)$ & 0.0001 \\
\hline Plaque length [mm (SD)] & $9.6(5.4)$ & $10.1(4.9)$ & 0.67 & $10.6(4.4)$ & $14.2(5.4)$ & 0.002 \\
\hline
\end{tabular}

ACS, acute coronary syndrome; SA, stable angina TCFA, thin cap fibroatheroma.

Vessel ulceration tended to occur without thrombus formation in patients with stable angina; in fact, only three ulcerated lesions were associated with thrombus. However, an accurate analysis of patients' clinical history revealed a recent (within 3-4 months) onset of anginal symptoms. Although these patients who exhibited ulcerated narrowing with thrombus did not meet the required clinical criteria to enter the unstable angina group ${ }^{5}$ at the time of the index procedure, they likely had a recent acute coronary event.

The present study showed that OCT can clarify morphological aspects of vessel angiographic haziness. In fact, in the intermediate stenosis group, the presence of vessel angiographic haziness was mostly associated with calcified plaques in stable angina and with thrombus in ACS. These findings not only confirm the distinctive aspects of stable angina and ACS in terms of plaque features, but also highlight how OCT can identify features that cannot be distinguished by angiography.

We finally showed that a strategy of percutaneous transluminal coronary angioplasty guidance, on the basis of the presence of coronary thrombus and/or a MLA less than $3.0 \mathrm{~mm}^{2}$, lead to a good outcome, with a low incidence of adverse clinical events at 12 months, comparable to that of patients with intermediate stenoses who were left on medical therapy only. Our group has provided the first evidence of the clinical utility of an OCT-guided PCI approach in patients with severe coronary stenoses. ${ }^{30}$

Although there is a need of larger prospective studies with long-term clinical follow-up, the present study shows the potential use of OCT as a pre-intervention guiding tool to select whether to treat culprit lesions in

Table 5 Events at 1-year follow-up in patients treated with percutaneous coronary intervention vs. optimal medical therapy

\begin{tabular}{lccc}
\hline & \multicolumn{3}{c}{ Intermediate lesion (56) } \\
\cline { 2 - 4 } & OMT (23) & PCl (33) & $P$ value \\
\hline Events at 1-year follow-up & & & \\
Cardiovascular death $[n(\%)]$ & $1(4)$ & $1(3)$ & 0.45 \\
Myocardial infarction $[n(\%)]$ & $1(4)$ & 0 & 0.18 \\
RR $[n(\%)]$ & $2(9)$ & $1(3)$ & 0.18 \\
Cardiac death, MI, RR $[n(\%)]$ & 0.26 \\
\hline
\end{tabular}

$\mathrm{MI}$, myocardial infarction; OMT, optimal medical therapy; $\mathrm{PCl}$, percutaneous coronary intervention; RR, repeated revascularization. addition to cardiac biomarkers, electrocardiographic changes, and angiography.

\section{Conclusion}

Intermediate coronary stenoses showed distinctive OCTbased features according to the initial clinical presentation. The adoption of an OCT-guided PCI strategy, on the basis of the presence of coronary thrombus and significant vessel narrowing, led to encouraging results. Despite the need for further large-scale studies, our results suggest the possible role of OCT to overcome the limits of coronary angiography for assessing intermediate stenosis and guiding primary treatment.

\section{Limitations}

The study lacks a sample size calculation, and we cannot exclude that a larger number of patients is needed to identify differences in terms of clinical outcome in patients with an intermediate stenosis treated with or without PCI on the basis of OCT findings. However, no study has previously studied the impact of MLA or thrombus detected by OCT in guiding PCI in a cohort of patients with a single de-novo intermediate stenosis.

\section{References}

1 Koo BK, Yang HM, Doh JH, et al. Optimal intravascular ultrasound criteria and their accuracy for defining the functional significance of intermediate coronary stenoses of different locations. JACC Cardiovasc Interv 2011; 4:803-811.

2 Prati F, Guagliumi G, Mintz GS, et al. Expert review document part 2: Methodology, terminology and clinical applications of optical coherence tomography for the assessment of interventional procedures. Eur Heart $J$ 2012; 33:2513-2520.

3 Tearney GJ, Regar E, Akasaka T, et al. Consensus standards for acquisition, measurement, and reporting of intravascular optical coherence tomography studies: A report from the international working group for intravascular optical coherence tomography standardization and validation. J Am Coll Cardiol 2012; 59:1058-1072.

4 Patel MR, Dehmer GJ, Hirshfeld JW, et al. Accf/scai/sts/aats/aha/asnc 2009 appropriateness criteria for coronary revascularization: A report of the american college of cardiology foundation appropriateness criteria task force, society for cardiovascular angiography and interventions, society of thoracic surgeons, american association for thoracic surgery, american heart association, and the american society of nuclear cardiology. Endorsed by the american society of echocardiography, the heart failure society of america, and the society of cardiovascular computed tomography. Catheter Cardiovasc Interv 2009; 73:E1-E24.

5 Fihn SD, Gardin JM, Abrams J, et al. 2012 accf/aha/acp/aats/pcna/scai/sts guideline for the diagnosis and management of patients with stable ischemic heart disease: a report of the american college of cardiology foundation/american heart association task force on practice guidelines, and the american college of physicians, american association for thoracic surgery, preventive cardiovascular nurses association, society for cardiovascular angiography and interventions, and society of thoracic surgeons. J Am Coll Cardiol 2012; 60:e44-e164. 
6 Jneid H, Anderson JL, Wright RS, et al. 2012 ACCF/AHA focused update of the guideline for the management of patients with unstable angina/nonstelevation myocardial infarction (updating the 2007 guideline and replacing the 2011 focused update): a report of the american college of cardiology foundation/american heart association task force on practice guidelines. J Am Coll Cardiol 2012; 60:645-681.

7 O'Gara PT, Kushner FG, Ascheim DD, et al. 2013 ACCF/AHA guideline for the management of st-elevation myocardial infarction: a report of the American College of Cardiology Foundation/American Heart Association task force on practice guidelines. J Am Coll Cardiol 2013; 61:e78-e140.

8 Garrone P, Biondi-Zoccai G, Salvetti I, et al. Quantitative coronary angiography in the current era: principles and applications. J Interv Cardiol 2009; 22:527-536.

9 Niccoli G, Giubilato S, Di Vito L, et al. Severity of coronary atherosclerosis in patients with a first acute coronary event: a diabetes paradox. Eur Heart $J$ 2013; 34:729-741.

10 Prati F, Regar E, Mintz GS, et al. Expert review document on methodology, terminology, and clinical applications of optical coherence tomography: PHYSICAL principles, methodology of image acquisition, and clinical application for assessment of coronary arteries and atherosclerosis. Eur Heart J 2010; 31:401-415.

11 Jang IK, Tearney GJ, MacNeill B, et al. In vivo characterization of coronary atherosclerotic plaque by use of optical coherence tomography. Circulation 2005; 111:1551-1555

12 Goto I, Itoh T, Kimura T, et al. Morphological and quantitative analysis of vascular wall and neointimal hyperplasia after coronary stenting: comparison of bare-metal and sirolimus-eluting stents using optical coherence tomography. Circ J 2011; 75:1633-1640.

13 Jia $H$, Abtahian $F$, Aguirre $A D$, et al. In vivo diagnosis of plaque erosion and calcified nodule in patients with acute coronary syndrome by intravascular optical coherence tomography. J Am Coll Cardiol 2013; 62:1748-1758.

14 Lee T, Yonetsu T, Koura K, et al. Impact of coronary plaque morphology assessed by optical coherence tomography on cardiac troponin elevation in patients with elective stent implantation. Circ Cardiovasc Interv 2011; 4:378-386.

15 Kitabata H, Tanaka A, Kubo T, et al. Relation of microchannel structure identified by optical coherence tomography to plaque vulnerability in patients with coronary artery disease. Am J Cardiol 2010; 105:16731678.

16 Imola F, Mallus MT, Ramazzotti V, et al. Safety and feasibility of frequency domain optical coherence tomography to guide decision making in percutaneous coronary intervention. Eurolntervention 2010; 6:575-581.
17 Thygesen K, Alpert JS, Jaffe AS, et al. Third universal definition of myocardial infarction. J Am Coll Cardiol 2012; 60:1581-1598.

18 De Bruyne B, Pijls NH, Kalesan B, et al. Fractional flow reserve-guided pci versus medical therapy in stable coronary disease. N Engl J Med 2012; 367:991-1001.

19 Pijls NH, van Schaardenburgh P, Manoharan G, et al. Percutaneous coronary intervention of functionally nonsignificant stenosis: 5-year followup of the defer study. J Am Coll Cardiol 2007; 49:2105-2111.

20 Tonino PA, De Bruyne B, Pijls NH, et al. Fractional flow reserve versus angiography for guiding percutaneous coronary intervention. $N$ Eng/ J Med 2009; 360:213-224.

21 Kern MJ, Samady H. Current concepts of integrated coronary physiology in the catheterization laboratory. J Am Coll Cardiol 2010; 55:173-185.

22 Little WC, Constantinescu M, Applegate RJ, et al. Can coronary angiography predict the site of a subsequent myocardial infarction in patients with mild-to-moderate coronary artery disease? Circulation 1988 78:1157-1166

23 Ambrose JA, Tannenbaum MA, Alexopoulos D, et al. Angiographic progression of coronary artery disease and the development of myocardial infarction. J Am Coll Cardiol 1988; 12:56-62.

24 Dacanay S, Kennedy HL, Uretz E, et al. Morphological and quantitative angiographic analyses of progression of coronary stenoses. A comparison of q-wave and nonq-wave myocardial infarction. Circulation 1994; 90:1739-1746.

25 Kotani J, Mintz GS, Castagna MT, et al. Intravascular ultrasound analysis of infarct-related and noninfarct-related arteries in patients who presented with an acute myocardial infarction. Circulation 2003; 107:2889-2893.

26 Fujii K, Kobayashi Y, Mintz GS, et al. Intravascular ultrasound assessment of ulcerated ruptured plaques: a comparison of culprit and nonculprit lesions of patients with acute coronary syndromes and lesions in patients without acute coronary syndromes. Circulation 2003; 108:2473-2478.

27 Kolodgie FD, Virmani R, Burke AP, et al. Pathologic assessment of the vulnerable human coronary plaque. Heart 2004; 90:1385-1391.

28 Kato K, Yonetsu T, Kim SJ, et al. Nonculprit plaques in patients with acute coronary syndromes have more vulnerable features compared with those with nonacute coronary syndromes: a 3-vessel optical coherence tomography study. Circ Cardiovasc Imaging 2012; 5:433-440.

29 Kato K, Yonetsu T, Jia H, et al. Nonculprit coronary plaque characteristics of chronic kidney disease. Circ Cardiovasc Imaging 2013; 6:448-456.

30 Prati F, Di Vito L, Biondi-Zoccai G, et al. Angiography alone versus angiography plus optical coherence tomography to guide decision-making during percutaneous coronary intervention: The centro per la lotta contro l'infarto-optimisation of percutaneous coronary intervention (cli-opci) study. Eurolntervention 2012; 8:823-829. 